\title{
Contact Angle and Surface Tension in Studies of Lung Surfactant
}

\author{
Hideo Sawada, Sumie Shioya, Yuji Takasaki, Koji Namba \\ and Hajime Yamabayashi \\ Department of Internal Medicine, School of Medicine, Tokai \\ University, Isehara 259-11
}

\begin{abstract}
Sawada, H., Shioya, S., Takasaki, Y., Namba, K. and Yamabayashi, H. Contact Angle and Surface Tension in Studies of Lung Surfactant. Tohoku J. exp. Med., 1978, $124(3), 233-240$ - The contact angle ( $(\theta)$ was determined in combination with surface tension measured in a Langmuiur-Willhelmy surface tension balance. Correction of the surface tension by the contact angle factor $(1 / \cos \theta)$ was tried on the monolayer curve of dipalmitoyl phosphatidyl choline (DPPC) and total lipid extracts from the bovine lung surfactant material (TLE). The contact angle was observed with an optical apparatus called a Contangulometer and pictures taken discontinuously, and was determined by drawing tangents at the peak of a junction between the water mensicus and a glass plate. The contact angle was about $15^{\circ}$, and had a narrow range of variation until the surface tension became very low, less than 5 dynes $/ \mathrm{cm}$, and then the contact angle jumped to almost $90^{\circ}$ both in DPPC and in TLE. It is concluded that the directly recorded surface tension curve underestimates the value by about $3-4 \%$ and that when the surface tension becomes very low, less than 5 dynes $/ \mathrm{cm}$, the value is no longer valid because the contact angle becomes nearly $90^{\circ}$. - contact angle; Langmuir-Wilhelmy surface tension balance; DPPC; TLE; lung surfactant
\end{abstract}

The surface tension balance was first applied to the investigation of pulmonary surfactant by Clements (1957), who employed a waxed Langmuir trough and a barrier with a Wilhelmy dipping plate sensor for surface tension and observed very low surface tension when the surface area was compressed. This and the subsequent studies have all used a value of zero for contact angle in surface tension measurements. Contact angle may increase with decreased surface tension in the presence of various surfactants. An apparatus was arranged to observe contact angle in combination with surface tension measurements. Contact angle can be measured from the pictures taken discontinuously, while surface area is changed with the barrier in a Langmuir trough. The present report describes an apparatus for contact angle measurement in combination with surface tension recordings of the monolayer of dipalmitoyl phosphatidyl choline (DPPC) and total lipid extracts from bovine lung surfactant (TLE) spread on water surface in Langmuir trough to try to check the validity of the idea that, "contact angle is zero." 


\section{Materials and Methods}

A Langmuir-Wilhelmy surface tension balance was constructed in our laboratory with the technical assistance by Nihon Kohden Co., Ltd. The trough was $700 \mathrm{~mm}$ in length, $200 \mathrm{~mm}$ wide and $50 \mathrm{~mm}$ in depth, made of stainless steel and coated by 3F-Teflon. The barrier, made of stainless steel and covered by a Teflon ribbon, was tightly fitted to the wall of the trough by cushions designed specially for this purpose. The machine was equipped with 5 changes of speed of the barrier movement for both compression and expansion. Only one speed of $1 \mathrm{~mm} / \mathrm{sec}$ during compression was used for the present experiment. Temperature in the trough was controlled by a thermostatically controlled circulating system and the present measurement were performed at $15^{\circ} \mathrm{C}$.

Contact angle was observed using an optical system (Contangulometer type CA-C, Kyowa Co., Ltd.). Photographs were taken discontinuously (36 times for one run) with a camera attached to this optical sytem. To obtain the contact angle, we drew tangents at the peak of a junction between water mensicus and a glass plate, measured the angle between two tangents, and divided it by the factor 2 . Pictures of the contact angle ideally should be taken with the optical axis horizontal and at the same level as the water surface. With instrumental limitations, the present measurements were performed with an angle $(a)$ of $12^{\circ}$ and the real contact angle $(\theta)$ was obtained by correcting the measured one $\left(\theta^{\prime}\right)$, by means of equation 1 .

$$
\tan \theta=\frac{\tan \theta^{\prime}}{\cos \alpha}
$$

A cover glass $(24 \times 60 \mathrm{~mm}$ with a thickness of $0.15 \mathrm{~mm})$ was used as a dipping plate, and was connected to a strain gauge electrical balance (FD-pick up, SD-1T-H, Nihon Kohden, Co., Ltd.). The plate was rinsed carefully with an alkali-ethanol solution before use and was washed with a 1:1 chloroform methanol solution after every run. The corrected surface tension $(\gamma)$ taking into consideration the contact angle $(\theta)$ was obtained by dividing the measured surface tension $\left(\gamma^{\prime}\right)$ by the factor $\cos \theta$ (Equation 2).

$$
\gamma=\frac{\gamma^{\prime}}{\cos \theta}
$$

The monolayer curve was recorded with an X-Y recorder (7004 B, X-Y recorder, Hewlett Packard Co., Ltd.), taking the abscissa as surface tension and the ordinate as area. Dimensions of the area are expressed as $\AA^{2} /$ molecule, both in DPPC and TLE, taking the molecular number of phospholipids into consideration. The area of the ordinate means the area occupied by phospholipids, not taking into account other lipids in spite of their existence in TLE.

DPPC (Sigma, MW 734.1) was used without further purification. A known amount of DPPC was dissolved in chloroform and was applied to the water surface. TLE from bovine lung surfactant was obtained by lipid extraction from bovine lung surfactant according to Folch's method (Folch et al. 1957). Bovine lung surfactant was purified from fresh bovine lung wash, following essentially Frosolono's method (Frosolono et al. 1970). Phospholipid quantitation was carried out by measuring the concentration of phosphorus in TLE following Eibl's method (Eibl and Lands 1969).

\section{RESULtS}

Table 1 lists contact angle $(\theta)$, the measured surface tension $\left(\gamma^{\prime}\right)$, and the corrected surface tension $(\gamma)$ of DPPC as a function of area occupied by a single molecule of DDPC. Contact angle is a mean of the 3 closest values among 5, measured by 5 different, well trained observers. These are plotted in Fig. 1. The directly recorded surface tension curve of DPPC monolayer showed about 70 dynes/ 
TABLE 1. Calculation of contact angle and correction of surface tension in the monolayer of $D P P C$. Measured contact angle $\left(\theta^{\prime}\right), \tan \theta$, corrected contact angle $(\theta), \cos \theta$, measured surface tension $\left(\gamma^{\prime}\right)$ and corrected surface tension $(\gamma)$ are listed as a function of area.

\begin{tabular}{|c|c|c|c|c|c|c|}
\hline$\underset{\left(\AA^{2} / \text { molecule }\right)}{\text { Area }}$ & $\begin{array}{l}\theta^{\prime} \\
\left(^{\circ}\right)\end{array}$ & $\tan \theta$ & $\begin{array}{c}\theta \\
\left({ }^{\circ}\right)\end{array}$ & $\cos \theta$ & $\begin{array}{c}\gamma^{\prime} \\
\text { (dynes/cm) }\end{array}$ & $\stackrel{\gamma}{(\text { dynes } / \mathrm{cm})}$ \\
\hline 136.0 & 13.2 & 0.240 & 13.5 & 0.972 & 70.0 & 72.0 \\
\hline 130.0 & 13.6 & 0.246 & 13.8 & 0.971 & 70.0 & 72.1 \\
\hline 120.0 & 13.6 & 0.246 & 13.8 & 0.971 & 70.0 & 72.1 \\
\hline 110.0 & 13.7 & 0.249 & 14.0 & 0.970 & 70.0 & 72.2 \\
\hline 100.0 & 14.1 & 0.257 & 14.4 & 0.969 & 70.0 & 72.2 \\
\hline 95.0 & 15.3 & 0.279 & 15.6 & 0.963 & 70.0 & 72.7 \\
\hline 90.0 & 16.5 & 0.303 & 16.9 & 0.957 & 70.0 & 73.1 \\
\hline 85.0 & 17.0 & 0.312 & 17.3 & 0.955 & 69.5 & 72.8 \\
\hline 80.0 & 15.9 & 0.291 & 16.2 & 0.960 & 68.9 & 71.8 \\
\hline 77.5 & 17.3 & 0.317 & 17.6 & 0.953 & 68.4 & 71.8 \\
\hline 75.0 & 15.4 & 0.281 & 15.7 & 0.963 & 67.4 & 70.0 \\
\hline 72.5 & 16.1 & 0.296 & 16.5 & 0.959 & 65.9 & 68.7 \\
\hline 70.0 & 16.2 & 0.297 & 16.5 & 0.959 & 63.5 & 66.2 \\
\hline 67.5 & 16.3 & 0.298 & 16.6 & 0.958 & 59.8 & 62.4 \\
\hline 65.0 & 12.3 & 0.223 & 12.6 & 0.976 & 53.5 & 54.8 \\
\hline 62.5 & 13.9 & 0.252 & 14. 1 & 0.970 & 44.5 & 45.9 \\
\hline 61.25 & 16.0 & 0.293 & 16.3 & 0.960 & 38.2 & 39.8 \\
\hline 60.0 & 16.5 & 0.303 & 16.9 & 0.957 & 32.0 & 33.4 \\
\hline 58.75 & 18.0 & 0.332 & 18.4 & 0.949 & 25.3 & 26.7 \\
\hline 57.5 & 18.0 & 0.332 & 18.4 & 0.949 & 19.2 & 20.0 \\
\hline 56.25 & 18.0 & 0.332 & 18.4 & 0.949 & 16.3 & 17.2 \\
\hline 55.0 & 20.0 & 0.372 & 20.4 & 0.937 & 14.2 & 15.2 \\
\hline 53.75 & 20.5 & 0.382 & 20.9 & 0.934 & 11.6 & 12.4 \\
\hline 52.5 & 20.5 & 0.382 & 20.9 & 0.934 & 7.4 & 7.9 \\
\hline 51.25 & 23. 0 & 0.434 & 23.5 & 0.917 & 4.6 & 5.0 \\
\hline 50.0 & 25.0 & 0.477 & 25.5 & 0.903 & 2.2 & 2.4 \\
\hline 48.75 & 28.0 & 0.544 & 28.6 & 0.878 & 1.5 & 1.7 \\
\hline 47.5 & 90.0 & & & & 1.0 & \\
\hline 45.0 & 90.0 & & & & 0.6 & \\
\hline 42.5 & 90.0 & & & & 0.5 & \\
\hline 40.0 & 90.0 & & & & 0.5 & \\
\hline 37.5 & 90.0 & & & & 0.5 & \\
\hline 35.0 & 90.0 & & & & 0.5 & \\
\hline 30.0 & 90.0 & & & & 0.4 & \\
\hline
\end{tabular}

cm when the film was largely expanded (expanded phase), then showed a gradual decrease of surface tension as the film was being compressed (intermediate phase) and decreased abruptly at the area range of $60-40 \AA^{2} /$ molecule (condensed phase). The contact angle was about $15^{\circ}$ with rather little change from the beginning of film compression to about $48 \AA^{2}$ /molecule and then tended to increase abruptly to reach $90^{\circ}$, at a time when the surface tension was lowered to almost 0 dyne/cm. The surface tension of the monolayer curve corrected by the contact angle factor (thin line in Fig. 1) showed an initial surface tension of about 72 dynes $/ \mathrm{cm}$, then changed corresponding to the decrease of the measured surface tension. However, when the measured surface tension became close to $0 \mathrm{dyne} / \mathrm{cm}$, the corrected surface tension had no significance any more, because contact angle at this range became very close to $90^{\circ}$ and the surface tension $0 / 0 \mathrm{dyne} / \mathrm{cm}$ is 


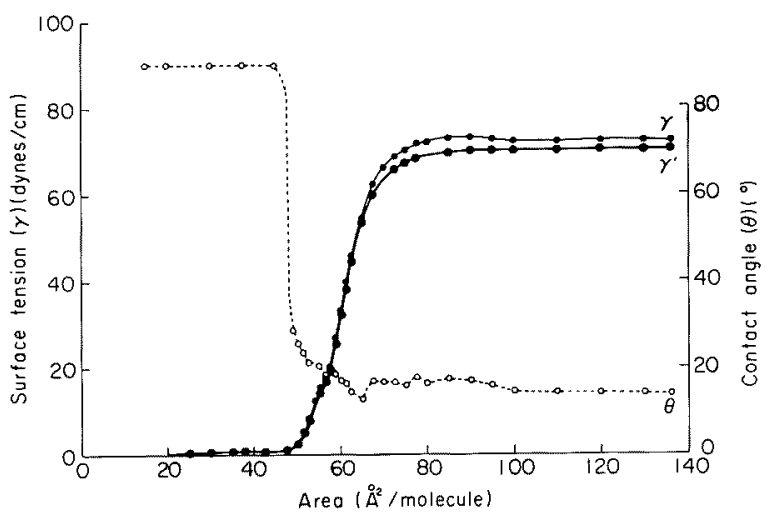

Fig. 1. Monolayer curve of DDPC. Directly recorded surface tension $\left(\gamma^{\prime}\right)$, contact angle $(\theta)$ and surface tension corrected by the factor of contact angle $(\gamma)$ are plotted against area occupied by single molecule of DPPC.

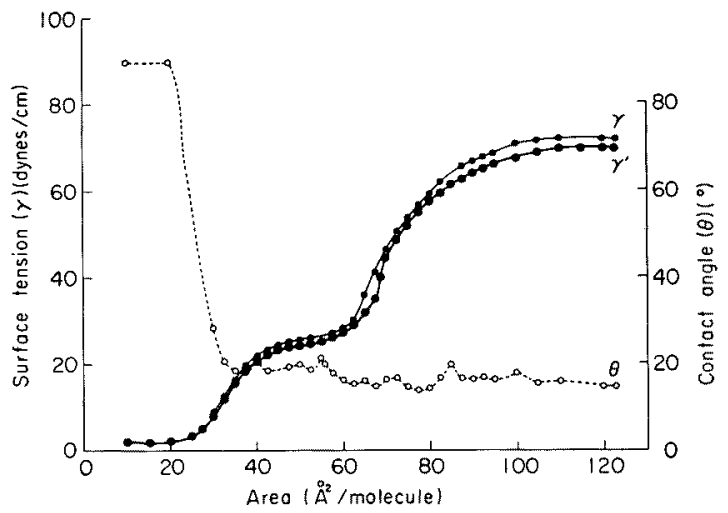

Fig. 2. Monolayer curve of TLE from bovine lung surfactant material. Ordinate is expressed as area occupied by a phospholipid molecule in TLE.

indeterminate.

The aspect of the monolayer curve of TLE (Fig. 2) is rather different from that of DPPC. From the beginning of film compression the surface tension tended to decrease gradually, showed two inflections at 70-60 $\AA^{2} /$ molecule and at 40-30 $\AA^{2} /$ molecule and then reached almost 0 dyne $/ \mathrm{cm}$. The contact angle showed the same tendency as that of DPPC; it kept a rather constant value of about $15^{\circ}$ until it was compressed to the area of $30 \AA^{2} /$ molecule and then jumped to almost $90^{\circ}$. Consequently, the corrected surface tension cannot be traced below $30 \AA^{2} /$ molecule (see Table 2 and Fig. 2).

\section{Discussron}

Although there is a statement that the contact angle should be zero when the surface tension is measured with Wilhelmy type dipping plate (Goerke 1974), no 
TABLE 2. Calculation of contact angle and correction of surface tension in the monolayer of $T L E$.

\begin{tabular}{|c|c|c|c|c|c|c|}
\hline $\begin{array}{c}\text { Area } \\
\left(\AA^{2} / \text { molecule }\right)\end{array}$ & $\begin{array}{l}\theta^{\prime} \\
\left({ }^{\circ}\right)\end{array}$ & $\tan \theta$ & $\begin{array}{c}\theta \\
\left(0^{\circ}\right)\end{array}$ & $\cos \gamma$ & $\begin{array}{c}\gamma^{\prime} \\
(\text { dynes/cm) }\end{array}$ & $\stackrel{\gamma}{(\text { dynes/m) }}$ \\
\hline 123.0 & 14.5 & 0.265 & 14.8 & 0.966 & 69.5 & 71.9 \\
\hline 120.0 & 14.2 & 0.258 & 14.5 & 0.968 & 69.5 & 71.8 \\
\hline 110.0 & 15.6 & 0.285 & 15.9 & 0.962 & 69.5 & 72.2 \\
\hline 105.0 & 15.1 & 0.276 & 15.4 & 0.964 & 68.8 & 71.4 \\
\hline 100.0 & 17.2 & 0.317 & 17.6 & 0.953 & 67.4 & 70.7 \\
\hline 95.0 & 15.8 & 0.290 & 16.2 & 0.960 & 65.8 & 68.5 \\
\hline 92.5 & 16.3 & 0.300 & 16.7 & 0.958 & 65.0 & 67.8 \\
\hline 90.0 & 16.0 & 0.294 & 16.4 & 0.959 & 63.9 & 66.6 \\
\hline 87.5 & 16.2 & 0.297 & 16.5 & 0.959 & 62.5 & 65.2 \\
\hline 85.0 & 19.5 & 0.362 & 19.9 & 0.940 & 61.4 & 65.3 \\
\hline 82.5 & 16.3 & 0.299 & 16.7 & 0.958 & 59.4 & 62.0 \\
\hline 80.0 & 13.9 & 0.252 & 14.2 & 0.969 & 57.4 & 59.2 \\
\hline 77.5 & 13.5 & 0.245 & 13.8 & 0.971 & 55.0 & 56.6 \\
\hline 75.0 & 14.3 & 0.261 & 14.6 & 0.968 & 52.0 & 53.7 \\
\hline 72.5 & 16.3 & 0.298 & 16.6 & 0.958 & 48.3 & 50.4 \\
\hline 70.0 & 16.1 & 0.295 & 16.4 & 0.959 & 44.5 & 46.4 \\
\hline 67.5 & 14.5 & 0.263 & 14.7 & 0.967 & 39.9 & 41.3 \\
\hline 65.0 & 15.7 & 0.286 & 16.0 & 0.961 & 34.5 & 35.9 \\
\hline 62.5 & 15.1 & 0.276 & 15.4 & 0.964 & 29.0 & 30.1 \\
\hline 60.0 & 16.0 & 0.294 & 16.4 & 0.959 & 27.2 & 28.4 \\
\hline 57.5 & 17.5 & 0.322 & 17.9 & 0.952 & 26.1 & 27.4 \\
\hline 55.0 & 20.8 & 0.389 & 21.3 & 0.932 & 25.3 & 27.1 \\
\hline 52.5 & 18.3 & 0.337 & 18.6 & 0.948 & 24.8 & 26.2 \\
\hline 50.0 & 19.4 & 0.361 & 19.8 & 0.941 & 24.2 & 25.7 \\
\hline 47.5 & 18.9 & 0.351 & 19.3 & 0.944 & 23.9 & 25.3 \\
\hline 45.0 & 18.4 & 0.340 & 18.8 & 0.947 & 23.2 & 24.5 \\
\hline 42.5 & 17.9 & 0.330 & 18.3 & 0.949 & 22.1 & 23.3 \\
\hline 40.0 & 20.9 & 0.389 & 21.3 & 0.932 & 20.5 & 22.0 \\
\hline 37.5 & 17.9 & 0.329 & 18.2 & 0.950 & 18.8 & 19.8 \\
\hline 35.0 & 18.1 & 0.334 & 18.5 & 0.949 & 15.5 & 16.3 \\
\hline 32.5 & 20.1 & 0.375 & 20.6 & 0.936 & 11.8 & 12.6 \\
\hline 30.0 & 27.9 & 0.541 & 28.4 & 0.880 & 7.9 & 9.0 \\
\hline 27.5 & Indeterminate & & & & 4.9 & \\
\hline 25.0 & Indeterminate & & & & 3.3 & \\
\hline 20.0 & 90.0 & & & & 2.1 & \\
\hline 15.0 & 90.0 & & & & 1.9 & \\
\hline 10.0 & 90.0 & & & & 1.9 & \\
\hline
\end{tabular}

one has ever measured the contact angle in combination with the surface tension measurement in the field of respiratory physiology. While we were preparing this manuscript, one paper (Hills and $\mathrm{Ng} 1974$ abstract) appeared in which the authors insisted that the contact angle can be more than zero and that it may influence the apparent increase of the hysteresis in the surface tension area curve. Our results of the contact angle measurement in combination with monolayer studies with a Langmuir-Wilhelmy surface tension balance shows about $15^{\circ}$ even when the monoalyer film is extremely expanded. The change of the contact angle is limited within a narrow range until the film is extremely compressed and consequently the surface tension becomes very close to $0 \mathrm{dyne} / \mathrm{cm}$ and then the contact angle jumps abruptly to $90^{\circ}$. When the surface pressure increases due to the film compression, the meniscus at the junction between the glass plate and 
water surface becomes smaller. The small area on the glass plate produced by the retraction of water meniscus may be coated by the surfactant material. The surface property of this area may change and cause the change of contact angle. The contact angle in our system is not an equilibrium one and the possible mechanism of the jump of contact angle from about $15^{\circ}$ to almost $90^{\circ}$ is in a rather complicated manner. It is beyond our description in the present paper and we would like to limit the description that this jump occured consistently under our experimental condition. Another thing to remember in this respect is that the monolayer film is extremely compressed at the point of the jump of contact angle and is in a condensed or in a solid phase; we are measuring the surface tension of the monolayer in its solid phase, slowly but continuously compressing it. A contact angle of $15^{\circ}$ causes a $3.4 \%$ underestimate of surface tension and in practice a check of the contact angle may not be necessary unless one intends to follow the surface tension to less than 5 dynes/cm, allowing an underestimate of $3-4 \%$. When the surface tension becomes less than 5 dynes/cm the contact angle suddenly jumps to almost $90^{\circ}$ and the surface tension cannot be traced effectively in this range with a Wilhelmy surface tension balance.

The cover glass we used as a dipping plate keeps some contact angle even when it is dipped into distilled water, although it is carefully rinsed before use and everytime before dipping it is rinsed with a 1:1 chloroform methanol solution in order to make it free from lipids which may be attached to the plate during the previous study. However, contact angle was checked in our measurement and in that sense we are convinced that the correction of surface tension by the contact angle factor leads us to a closer value to the absolute of surface tension.

The measurement of contact angle is accompanied by some errors. In the present experiment, we took picture (Fig. 3) in sequence and this enabled us to determine the contact angle several times on the same material, using well trained observers. In spite of this precaution, errors can be involved and induce some deviation on the value of the corrected surface tension. A device should be made for more precise and continuous measurement of the contact angle. In the present measurement the angle between the optical axis and water surface was taken into account and a correction was made according to equation (1) (see Appendix). The correction factor is very close to 1 and for practical purpose it is not always necessary.

In our monolayer study on TLE, the abscissa is arbitrarily shown in $\AA^{2}$ / molecule, taking account only of the phospholipids in TLE. It is quite arbitrary and for the convenience of comparison with DPPC. The monolayer of TLE shows two distinct inflections at 70-60 $\AA^{2} /$ molecule and at 40-32 $\AA^{2} /$ molecule, having an intermediate plateau between them. The contact angle at the inflection point of $40-30 \AA^{2} /$ molecule is about $20^{\circ}$. Correction of surface tension by the factor of contact angle at this range is possible and the corrected surface tension is valid. This means that the inflection at this range is significant and suggests that the monolayer of TLE has two condensing phases and that TLE contains two compounds or 


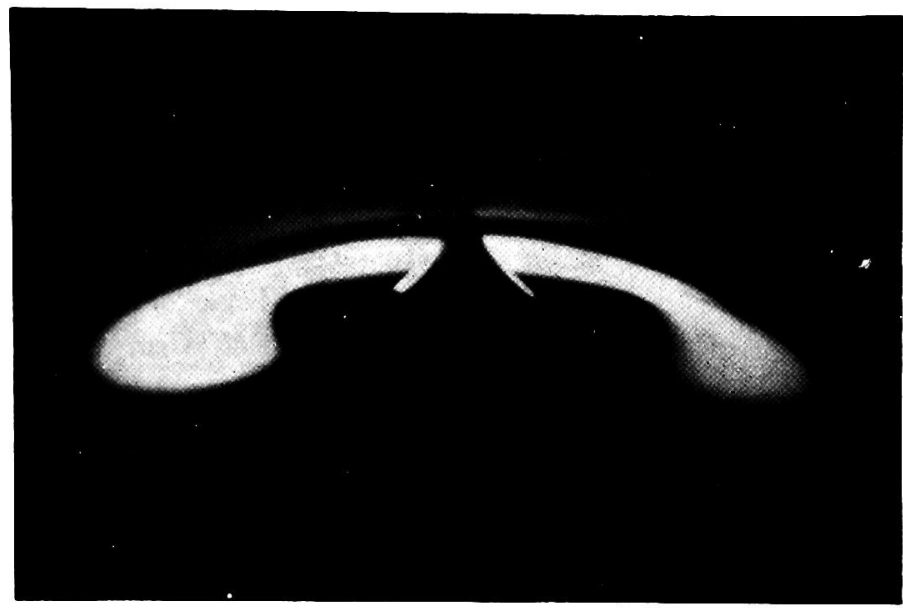

Fig. 3. Meniscus formed between water surface and a glass plate. Picture was taken during compression of the monolayer of TLE at the area of $120.0 \AA^{2}$ molecule. See text for the determination of contact angle.

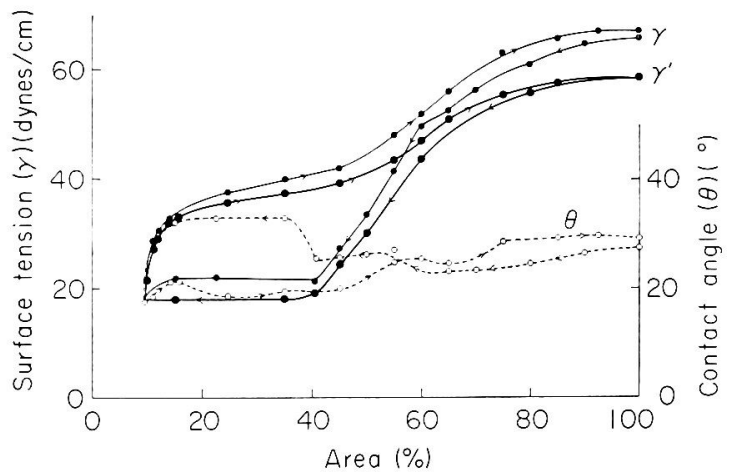

Fig. 4. Surface tension-area curve ( $\gamma$-A curve) of bovine lung surfactant material, measured in conventional way. Contact angle during decompression has a lower value than during compression and hysteresis of contact angle is observed in the area less than $50 \%$.

groups of compounds which will affect the monolayer behavior. Of course this remains speculative for the moment, because the monolayer curve was obtained at a single speed of compression; when the barrier stops on the way, the surface tension increases to some extent and the possibility remains that a different compression speed would make a different curve.

Hills and $\mathrm{Ng}$ (1947) reported a significantly thinner hysteresis loop of the surface tension vs. area curve when the ring method was used than when the Wilhelmy surface tension balance was used. In order to check this point, a small amount of bovine surfactant material, isolated by Frosolono's method (Frosolono et al. 1970), was suspended in distilled water and the surface tension area curve was 
recorded in conventional way. The contact angle was monitored by taking pictures at 28 points covering both the compression and decompression phases. Fig. 4 shows contact angle, monitored by taking pictures at 28 points covering both the compression and decompression phases after $\gamma$-A curve became stable. The change of contact angle also showed a hysteresis in the area range less than $50 \%$ (higher contact angle during compression than during decompression) and consequently the $\gamma$-A curve corrected by the factor of contact angle showed a thinner hysteresis than the directly recorded one. The higher contact angle of about $27-28^{\circ}$, even when the area was $100 \%$ distended, was probably due to adsorption of surfactant material on the glass plate during the sweep prior to the actual recording.

Appendix

In a triangular prism consisting of two tangential planes drawn at the junction between the fluid meniscus and glass plate, two triangles can be drawn, one perpendicular to the optical axis of Contangulometer and another one vertical to the water surface. From the parameters of two triangles shown in Fig. 5, the following 3 equations are obtained.

$$
\begin{aligned}
& \tan \theta=a / 2 h \\
& \tan \alpha=h / h^{\prime} \\
& \tan \theta^{\prime}=a / 2 h^{\prime}
\end{aligned}
$$

thus, from equation $\left(1^{\prime}\right),\left(2^{\prime}\right)$ and $\left(3^{\prime}\right)$, Equation $(1)$ is derived.

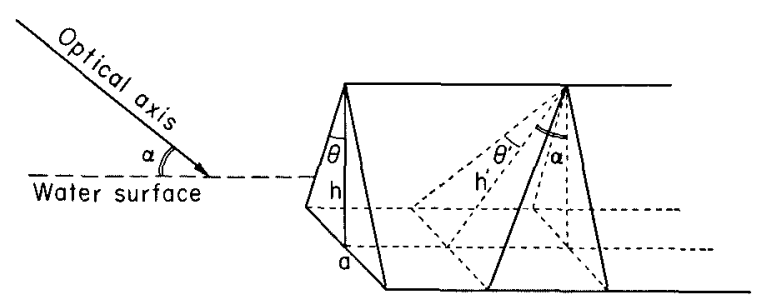

Fig. 5. Correction of contact angle due to the angle between the optical axis of Contangulometer and water surface in the trough $(\alpha)$. See Appendix.

\section{References}

1) Clements, J.A. (1957) Surface tension of lung extracts. Proc. Soc. exp. Biol. Med. (N.Y.), 95, 170-172.

2) Eibl, H. \& Lands, W.E.M. (1969) A new sensitive determination of phosphate. Analyt. Biochem., 30, 51-57.

3) Folch, J., Lees, M. \& Sloane Stanley, G.H. (1957) A simple method for the isolation and purification of total lipids from animal tissues. J. biol. Chem., 226, 497-509.

4) Frosolono, M.F., Charms, B.L., Pawlowski, R. \& Silvka, S. (1970) Isolation, characterization and surface chemistry of a surface active fraction from dog lung. J. Lipid, Res., 11, 439-457.

5) Goerke, J. (1974) Lung surfactant. Biochem. biophys. Acta (Amst.), 344, 241-261.

6) Hills, B.A. \& Ng, Y.L. (1974) Significance of the contact angle in studies of lung surfactant. J. Physiol. (Lond.), 241, 52-53. 